\title{
Impact of patient characteristics on the risk of influenza/ILI-related complications
}

\author{
Debra E Irwin ${ }^{1}$, Lisa B Weatherby ${ }^{1}$, Wen-Yi Huang ${ }^{2,3}$, \\ Daniel M Rosenberg ${ }^{4}$, Suzanne F Cook ${ }^{2}$ and Alexander M Walker*1
}

Address: ${ }^{1}$ Ingenix Pharmaceutical Services, Epidemiology Division, Newton Lower Falls, Massachusetts, USA, ${ }^{2}$ Worldwide Epidemiology, GlaxoSmithKline, Research Triangle Park, North Carolina, USA, ${ }^{3}$ Division of Cancer Epidemiology and Genetics, National Cancer Institute, Bethesda, Maryland, USA and 4Worldwide Epidemiology, GlaxoSmithKline, Greenford, UK

E-mail: Debra E Irwin - debra.irwin@ingenixPS.com; Lisa B Weatherby - lweatherby@epidemiology.com; Wen-

Yi Huang - huangw@mail.nih.gov; Daniel M Rosenberg - dr74409@glaxowellcome.co.uk; Suzanne F Cook - sfc3705@glaxowellcome.com; Alexander M Walker* - AlecWalker@Epidemiology.com

*Corresponding author

Published: 2I August 200I

BMC Health Services Research 200 I, I:8
Received: II June 200 I

Accepted: 21 August 200I

This article is available from: http://www.biomedcentral.com/I472-6963/I/8

(c) 200I Irwin et al; licensee BioMed Central Ltd. Verbatim copying and redistribution of this article are permitted in any medium for any non-commercial purpose, provided this notice is preserved along with the article's original URL. For commercial use, contact info@biomedcentral.com

\begin{abstract}
Background: We sought to quantify the impact of patient characteristics on complications and health care costs associated with influenza and influenza-like illness (ILI) in a nonelderly population.

Methods: Patients with medical reimbursement claims for influenza in the 1996-1997 season were identified from the automated database of a large private New England Insurer (NEI). Influenza care during the $2 \mathrm{I}$ - day follow-up period was characterized according to age, gender, vaccine status, co-morbidities, prior influenza/LI episodes, treatments, and recent health care costs and related diagnoses.
\end{abstract}

Results: There were 6,241 patients. Approximately $20 \%$ had preexisting chronic lung disease. Overall, $23 \%$ had health care services for possible complications, among which respiratory diagnoses were the most common (13\%). Two percent of the influenza/ILI episodes involved hospitalization, with a median stay of five days. Factors most strongly predictive of hospitalizations and complications were preexisting malignancy (hospitalizations $O R=3.7$ and complications $O R=$ 2.4), chronic heart disease $(O R=3.2$ and $O R=1.8)$, diabetes $(O R=2.2$ and $O R=1.7)$ and recent illnesses that would have counted as complications had they occurred during an influenza/ILI episode (hospitalizations $O R=3.2$ and complications $O R=1.5$ ). The same factors affected influenza-related costs and total costs of care as dramatically as they affected complication rates.

Conclusions: Influenza/ILI-related costs are driven by the characteristics that predict complications of influenza. Patients with chronic illness and those with recent acute respiratory events are the most likely to experience complications and hospitalizations.

\section{Background}

Influenza costs the US some 40,000 deaths, 200,000 hospitalizations, and millions of workdays lost each year.
[1-3] Most deaths (6o-90\%) due to influenza occur in patients 65 years and older, but $45-77 \%$ of influenza-related hospitalizations occur in patients younger than 65 
years old $[2,4,5]$. High complication rates from influenza in the elderly have also been reported [6,7]. Little is known about influenza-related morbidity and health care costs in younger populations and among those with preexisting conditions such as diabetes, cardiovascular, or pulmonary disease.

Diagnosis of influenza is typically based on the presence of symptoms and the temporal association with known circulating virus. At the time covered by this study, a rapid clinic-based diagnostic test was not available. Most research performed in an observational setting utilizes clinical diagnosis and therefore, includes both influenza and some influenza-like illnesses (ILI). A clinic-based study inevitably misses influenza that does not come to clinical attention. This study therefore provides data on the burden to the health payor of influenza, ILI, and their related complications in a population of 2.6 million children and adults with various risk profiles. The primary objective of this study was to examine the role of patient characteristics that might be predictive of complications and related health care costs associated with influenza and ILI.

\section{Methods}

\section{Data source}

We used the automated claims database of a large private New England Insurer (NEI). The NEI database contains medical claims for outpatient and inpatient services and outpatient pharmacy claims for about 2,600,00o residents of New England with comprehensive medical and prescription drug coverage. The number of people over age 65 is limited, since Medicare beneficiaries do not participate in NEI.

Each medical claim contains primary and secondary diagnoses codes assigned using the International Classification of Diseases, ninth Revision (ICD-9) [8]. Up to five procedure codes are also present on the claim records and are coded using ICD-9, Current Procedural Terminology (CPT), or Health Care Finance Administration (HCFA) codes. [8-10] Prescription drugs dispensed are identified by National Drug Code (NDC), American Hospital Formulary Service (AHFS) therapeutic category, brand name, and chemical name [11]. The inpatient transactions contain each service the provider listed on the claims for reimbursement, such as surgeries, radiologic tests, laboratory tests, room and board charges, or other specific cost items. Inpatient pharmacy costs are included in the aggregate hospital charges, and cannot be separated out. Charges by the provider and amounts paid by the insurer, patient, or other third parties are included.

\section{Study population}

Patients with a billed diagnosis of influenza (medical claim with either a primary or secondary ICD-9 diagnosis code of 487) meeting the following criteria were designated as having an influenza/ILI episode for the ensuing 21 days: 1 ) diagnosis occurred between the beginning of October 1996 and the end of April 1997; 2) diagnosis did not occur on the date of a immunization for influenza; 3) diagnosis was preceded by at least one year of continuous health insurance coverage, with no gaps in coverage that exceeded 30 days; and 4) insurance coverage during the 21-day follow up period was continuous. We will use the term "interval of care" interchangeably with "episode" to describe this period. Multiple claims for care of influenza for the same patient occurring within the 21-day follow-up period were taken to be part of a single episode. We refer to the qualifying influenza diagnosis day and the subsequent 21-day follow-up period as the interval of care throughout this report. The 21-day period captures the longest likely duration of symptoms directly caused by the influenza infection (7-10 days) plus a period to account for the evolution and resolution of complications of the infection. While a rare, serious complication could lead to a protracted illness, we felt that the problem of resolving the relative contributions of underlying and acute disease in these settings would introduce substantial error, and provide relatively little additional information. We will use the term "influenza/ ILI" throughout this manuscript recognizing that medical claims with an ICD-9 code of 487 include influenza as well as some cases of ILI.

\section{Characteristics of influenza/lLI-related episodes}

We characterized each influenza/ILI episode according to: (1) age at time of influenza/ILI diagnosis; (2) gender; (3) recorded influenza immunization status; (4) prior influenza/ILI episodes during the study period; (5) antiviral influenza treatment (amantadine or rimantadine); (6) total patient health care costs in the three months before influenza/ILI diagnosis); (7) co-morbidities for which health services were received in (a) the 30 days and (b) the year before influenza/ILI diagnosis (chronic lung disease, chronic renal disease, diabetes, malignancies, HIV infections, and chronic heart disease); (8) immunosuppressive and chronic corticosteroid drug use during the year before influenza/ILI diagnosis; (9) health services in the 30 days before influenza/ILI diagnosis for conditions that would have been characterized as complications or manifestations had they occurred during the influenza/ILI episode (see below). The 30day window was chosen on clinical grounds to capture medical conditions that were unstable, and the one-year window was intended to capture all active medical conditions. Inevitably, some routine care will have occurred in the 30-day window, and some conditions treated in 
the past year will not have been active at the time of the influenza episode.

We evaluated influenza immunizations between September 1, 1996 and the day before the initial influenza/ILI diagnosis. Antiviral influenza medications dispensed within the three days before, the day of, or the three days after the initial influenza/ILI diagnosis date were noted. We created this seven-day time window to account for prescriptions that may have been dispensed for the current diagnosis before an office visit (e.g., by telephoned prescription) or within a few days after an office visit. We categorized health care costs in the three months before the influenza/ILI diagnosis in quintile ranges as follows: (1) $\$ 0$, (2) up to $\$ 87$, (3) $\$ 87-\$ 200$, (4) $\$ 200-\$ 453$ and (5) $\$ 453$ and above. The mean and median threemonth costs preceding influenza/ILI diagnoses was $\$ 644$ and $\$ 135$, respectively. The underlying co-morbid conditions we considered included chronic lung disease, chronic renal disease, diabetes, malignancies, HIV infections, chronic heart disease, and chronic renal disease.

\section{Complications and manifestations of influenza/ILI}

We defined complications and manifestations occurring on the day of the influenza/ILI diagnosis or during the 21-day follow-up period using individual or combinations of ICD-9 codes to capture respiratory, cardiovascular, infectious, musculoskeletal, symptoms, and other diseases. Ambulatory care visits, hospitalizations, procedures, and emergency room visits were included. Throughout this report, these are referred to as complications of influenza/ILI. Please see Additional File 1[Appendix A.doc] for specific ICD9 codes.

\section{Comorbidities}

We identified the presence of chronic lung disease, chronic renal disease, diabetes, malignancies, HIV infections, and chronic heart disease using algorithms based on health services utilization, detailed in Additional File 1[Appendix B.doc].

\section{Hospitalizations}

Influenza/ILI-related hospitalizations were defined using the ICD-9 diagnoses codes recorded on the detailed inpatient claims, categorized as listed above. We calculated mean and median length of stay in hospital. There were 10 (out of 115) hospitalizations that continued beyond the 21-day follow-up period. For the complications analysis, these counted in exactly the same manner as the 105 hospitalizations that were completed within the 21-day follow-up. For the primary cost and duration of stay analyses data from the 10 extended hospitalizations were retained only for hospitalized days in the follow-up period, as an approximate way of partitioning costs for events with mulitfactorial etiology. Total hospital cost was multiplied by the proportion of hospital days that fell within the follow-up period. We also present the total cost and length of stay without truncation.

\section{Health care costs}

We characterized each influenza/ILI interval of care by total cost and influenza/ILI-related cost. We defined influenza/ILI-related costs as those associated with ongoing care for influenza/ILI and with complications of influenza/ILI, including medication costs, which occurred during the influenza/ILI interval of care (see above list of ICD-9 codes). Influenza/ILI-related medications were those commonly prescribed for influenza/ ILI symptoms, manifestations and complications (oral, injectable, ophthalmic and topical antibiotics; antitussives; expectorants, and mucolytic agents; decongestants;aspirin, acetaminophen, ibuprofen, naproxen; amantadine, rimantadine; beta-agonists; theophylline; oral, injectable, rectal or inhaled steroids; ipratropium and cromolyn sodium).

We defined total costs as the sum of total health care costs for all services incurred during the interval of care. Costs presented in this report were the sum of the amount paid by the insurer and the patient. Patient costs include a patient's deductible, co-payment, coinsurance, and coordination of benefit amounts. Societal costs and patient level costs such as lost workdays were not considered.

\section{Analysis}

We used logistic regression modeling to examine the association of potential predictive factors with influenza/ ILI-related complications and hospitalizations. These models permitted adjustment for all of the possible predictive factors concurrently; no variables were dropped from the models.

\section{Results}

\section{Study population and intervals of care}

6,269 persons met eligibility and enrollment criteria; they experienced 6,660 intervals of influenza/ILI care. Thirty intervals of care were excluded from the analysis because they occurred in subgroups with very small numbers of patients, within which it would have been impossible to obtain stable disease-specific estimates (HIV (4 men; 2 women); chronic renal disease (5 men; 7 women) and immunosuppressive therapy (3 men; 10 women)). The remaining 6,630 influenza/ILI intervals of care $(6,241$ patients) formed the basis of this analysis.

Ninety-four percent of patients $(5,895)$ had a single episode. Approximately $5 \%$ (308) experienced two episodes and $1 \%(30)$ had three or more during the study period. In $94 \%$ of the intervals of care, influenza/ILI was diag- 
nosed in an outpatient setting. Approximately 5\% were diagnosed in the emergency room and $1 \%$ were diagnosed upon hospitalization.

Approximately $38 \%$ of episodes included outpatient procedures or services associated with influenza/ILI or complications. Outpatient dispensings were recorded for $50 \%$ of the episodes for the medications commonly prescribed for influenza/ILI symptoms, manifestations, and complications on the day of or during the 21 days beyond their diagnosis. Antibiotics were prescribed for 39\% of the episodes. Four percent of the episodes had dispensings of antivirals (amantadine or rimantadine).
Overall, $23 \%$ of the influenza/ILI episodes had evidence of one or more co-morbid conditions occurring in the year before the influenza/ILI diagnosis. Chronic lung disease was the most prevalent, observed for approximately $20 \%$ of the influenza/ILI episodes, at every age. Chronic corticosteroid use, malignancy, chronic heart disease, and diabetes became more common with advancing age. In approximately $2 \%$ of the influenza/ILI intervals of care, patients had evidence of multiple comorbidities and this rose with higher age groups, reaching $21 \%$ for males age $65^{+}$and $17 \%$ for females age $65^{+}$. (Table 1).

Table I: Prevalence of Medication Use and Comorbidities in the Year Before the Influenza Diagnosis

\begin{tabular}{|c|c|c|c|c|c|c|c|c|c|c|c|c|c|c|c|}
\hline $\begin{array}{l}\text { Age (years) } \\
\text { and Gender }\end{array}$ & Episodes & $\begin{array}{l}\text { Chronic } \\
\text { Corticosteroid }\end{array}$ & & Malignancy & & $\begin{array}{l}\text { Chronic } \\
\text { Heart } \\
\text { Disease }\end{array}$ & & $\begin{array}{l}\text { Chronic } \\
\text { Lung } \\
\text { Disease }\end{array}$ & & Diabetes & & Multiple & & Any & \\
\hline & $N$ & $\mathrm{~N}$ & $\%$ & $N$ & $\%$ & $\mathrm{~N}$ & $\%$ & $N$ & $\%$ & $N$ & $\%$ & $N$ & $\%$ & $N$ & $\%$ \\
\hline \multicolumn{16}{|l|}{$0-4$} \\
\hline Male & 648 & 1 & 0 & 0 & 0 & 1 & 0 & 142 & 22 & 0 & 0 & 0 & 0 & 144 & 22 \\
\hline Female & 573 & 1 & 0 & 0 & 0 & 1 & 0 & 100 & 17 & 0 & 0 & 2 & 0 & 100 & 17 \\
\hline \multicolumn{16}{|l|}{$5-14$} \\
\hline Male & 806 & 0 & 0 & I & 0 & 0 & 0 & 185 & 23 & I & 0 & 1 & 0 & 186 & 23 \\
\hline Female & 826 & 1 & 0 & 2 & 0 & 1 & 0 & 173 & 21 & 0 & 0 & I & 0 & 176 & 21 \\
\hline \multicolumn{16}{|l|}{$15-24$} \\
\hline Male & 253 & 0 & 0 & I & 0 & 0 & 0 & 44 & 17 & 0 & 0 & 0 & 0 & 45 & 18 \\
\hline Female & 336 & 1 & 0 & 0 & 0 & 0 & 0 & 73 & 22 & 1 & 0 & 0 & 0 & 75 & 22 \\
\hline \multicolumn{16}{|l|}{$25-34$} \\
\hline Male & 284 & 0 & 0 & 1 & 0 & 0 & 0 & 39 & 14 & I & 0 & I & 0 & 40 & 14 \\
\hline Female & 445 & 1 & 0 & 0 & 0 & 1 & 0 & 72 & 16 & 5 & 1 & 4 & 1 & 75 & 17 \\
\hline \multicolumn{16}{|l|}{$35-44$} \\
\hline Male & 422 & 3 & 1 & 5 & 1 & 8 & 2 & 59 & 14 & 8 & 2 & 7 & 2 & 74 & 18 \\
\hline Female & 553 & 3 & 1 & 6 & 1 & 2 & 0 & 98 & 18 & 9 & 2 & 5 & 1 & 113 & 20 \\
\hline \multicolumn{16}{|l|}{$45-54$} \\
\hline Male & 356 & 2 & I & 6 & 2 & 19 & 5 & 67 & 19 & 18 & 5 & 16 & 4 & 94 & 26 \\
\hline Female & 450 & 3 & I & 15 & 3 & 9 & 2 & 90 & 20 & 10 & 2 & 12 & 3 & 113 & 25 \\
\hline \multicolumn{16}{|l|}{$55-64$} \\
\hline Male & 209 & 2 & I & 6 & 3 & 32 & 15 & 39 & 19 & 18 & 9 & 16 & 8 & 77 & 37 \\
\hline Female & 299 & 6 & 2 & 14 & 5 & 13 & 4 & 66 & 22 & 24 & 8 & 17 & 6 & 99 & 33 \\
\hline \multicolumn{16}{|l|}{$65+$} \\
\hline Male & 57 & 1 & 2 & 7 & 12 & 16 & 28 & 11 & 19 & 8 & 1 & 12 & 2 & 29 & 51 \\
\hline & & & & & & & & & & & 4 & & 1 & & \\
\hline Female & 113 & 4 & 4 & 8 & 7 & 25 & 22 & 25 & 22 & 13 & 1 & 19 & 1 & 51 & 45 \\
\hline & & & & & & & & & & & 2 & & 7 & & \\
\hline
\end{tabular}

\begin{tabular}{|c|c|c|c|c|c|c|c|c|c|c|c|c|c|c|c|}
\hline Total & & & & & & & & & & & & & & & \\
\hline Male & 3,035 & 9 & 0 & 27 & I & 76 & 3 & 586 & 19 & 54 & 2 & 53 & 2 & 689 & 23 \\
\hline Female & 3,595 & 20 & 1 & 45 & I & 52 & 1 & 697 & 19 & 62 & 2 & 60 & 2 & 802 & 22 \\
\hline
\end{tabular}


Table 2: Predictors of Influenza-Related Complications and Hospitalizations

Type of Complication

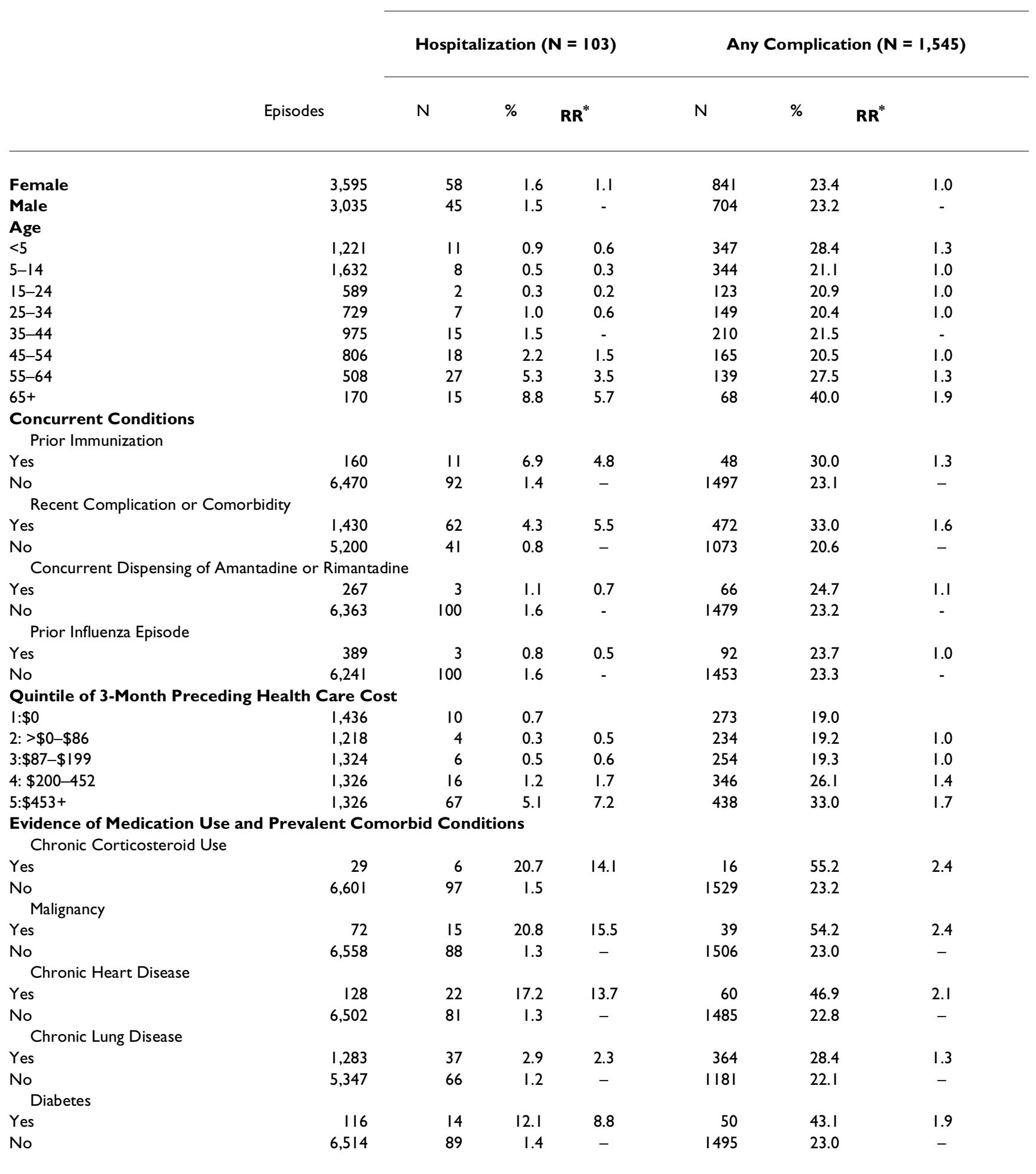




\begin{tabular}{|c|c|c|c|c|}
\hline & & Presen & ring Interva & \\
\hline & Hos & $v=(03)$ & & $(\mathbf{N}=1,545)$ \\
\hline & $O \mathbf{R}^{*}$ & $95 \% \mathrm{Cl}$ & OR* & $95 \% \mathrm{Cl}$ \\
\hline Sex (Female vs. Male) & 1.02 & $0.67-1.56$ & 1.00 & $0.89-1.12$ \\
\hline Age & & & & \\
\hline$<5$ & 0.75 & $0.33-1.70$ & 1.55 & $1.26-1.91$ \\
\hline $5-14$ & 0.45 & $0.19-1.10$ & 1.08 & $0.89-1.32$ \\
\hline $15-24$ & 0.28 & $0.06-1.26$ & 1.02 & $0.79-1.31$ \\
\hline $25-34$ & 0.83 & $0.33-2.07$ & 0.99 & $0.78-1.26$ \\
\hline $35-44$ & 1.00 & $\mathrm{n} / \mathrm{a}$ & 1.00 & \\
\hline $45-54$ & 1.22 & $0.59-2.52$ & 0.85 & $0.67-1.08$ \\
\hline $55-64$ & $\mathrm{I} .75$ & $0.87-3.52$ & 1.04 & $0.80-1.35$ \\
\hline $65+$ & 1.93 & $0.82-4.56$ & 1.54 & $1.06-2.24$ \\
\hline Prior/Concurrent & & & & \\
\hline Prior Immunization & 2.17 & $1.06-4.43$ & 1.01 & $0.70-1.45$ \\
\hline Recent Comp/Comorbidity & 3.17 & $1.96-5.13$ & 1.47 & $1.27-1.70$ \\
\hline Concurrent Amant/ Rimant & 0.60 & $0.18-2.00$ & 1.22 & $0.91-1.63$ \\
\hline Prior Influenza Episode & 0.32 & $0.09-1.07$ & 0.85 & $0.66-1.09$ \\
\hline Quintile of 3-Month Precedi & Care Cost & & & \\
\hline $\mathrm{I}: \$ 0$ & 1.00 & & 1.00 & \\
\hline $2:>\$ 0-\$ 86$ & 0.38 & $0.22-1.22$ & 0.92 & $0.75-1.12$ \\
\hline $3: \$ 87-\$ 199$ & 0.44 & $0.15-1.24$ & 0.86 & $0.70-1.04$ \\
\hline 4: $\$ 200-452$ & 0.84 & $0.36-1.97$ & 1.21 & $1.00-1.47$ \\
\hline $5: \$ 453+$ & I.7I & $0.77-3.84$ & 1.56 & $1.28-1.91$ \\
\hline Medication Use and Prevale & bid Conditior & & & \\
\hline Chronic Corticosteroid Use & 2.00 & $0.66-6.02$ & 1.84 & $0.83-4.07$ \\
\hline Malignancy & 3.67 & I.82-7.44 & 2.38 & $1.44-3.93$ \\
\hline Chronic Heart Disease & 3.16 & $1.66-6.00$ & 1.76 & $1.19-2.6 \mathrm{I}$ \\
\hline Chronic Lung Disease & 1.29 & $0.8 I-2.05$ & 1.15 & $1.00-1.34$ \\
\hline Diabetes & 2.19 & $1.08-4.47$ & 1.74 & $1.16-2.6 \mid$ \\
\hline
\end{tabular}

* Adjusted using logistic regression.

\section{Influenza/ILI-related complications and hospitalizations} Overall, 23\% of intervals of care had an influenza/ILI-related complication. Respiratory complications were the most common (13\%). Approximately $2 \%$ of influenza/ILI intervals of care resulted in a hospitalization for influenza/ILI-related complications. Four percent resulted in an emergency room visit and $20 \%$ resulted in an office visit (data not shown).

The median and mean length of hospital stay during the observation period for influenza/ILI-related complications was 5 days. Including portions of hospitalizations that extended outside of the 21-day observation period, the median length of stay in hospital was still five days. The mean was 8.4 days.
Table 2 shows the unadjusted associations for the potential predictors of influenza/ILI-related hospitalizations and complications during the influenza/ILI intervals of care. In general, the patterns seen for factors associated with hospitalizations were similar to those seen for influenza/ILI-related complications. Older age, prior immunization, recent complications or co-morbid diagnoses (30 days before the influenza/ILI diagnosis), co-morbidities identified in the year prior to diagnosis, and high recent health care costs (in the previous three months) were all strongly associated with hospitalizations or complications of influenza/ILI.

Table 3 presents the results from the logistic regression analyses for predictors of any type of influenza/ILI-related complication and hospitalization. Adults in the oldest 
Table 4: Predictors of Costs Related to Influenza Complications and of Total Health Care Costs

\begin{tabular}{|c|c|c|c|c|}
\hline & \multicolumn{2}{|c|}{ Influenza-Related Costs } & \multicolumn{2}{|c|}{ Total Costs } \\
\hline & Mean & Median & Mean & Median \\
\hline \multicolumn{5}{|l|}{ Gender } \\
\hline Female & $\$ 303$ & $\$ 69$ & $\$ 409$ & $\$ 107$ \\
\hline Male & $\$ 294$ & $\$ 67$ & $\$ 380$ & $\$ 93$ \\
\hline \multicolumn{5}{|l|}{ Age } \\
\hline $0-4$ & $\$ 150$ & $\$ 62$ & $\$ 184$ & $\$ 83$ \\
\hline $5-14$ & $\$ 111$ & $\$ 61$ & $\$ 152$ & $\$ 69$ \\
\hline 15-24 & $\$ 125$ & $\$ 69$ & $\$ 184$ & $\$ 100$ \\
\hline $25-34$ & $\$ 211$ & $\$ 68$ & $\$ 339$ & $\$ 103$ \\
\hline $35-44$ & $\$ 335$ & $\$ 77$ & $\$ 425$ & $\$ 118$ \\
\hline $45-54$ & $\$ 639$ & $\$ 80$ & $\$ 811$ & $\$ 14 \mid$ \\
\hline $55-64$ & $\$ 762$ & $\$ 93$ & $\$ 959$ & $\$ 182$ \\
\hline $65+$ & $\$ 944$ & $\$ 109$ & $\$ 1,408$ & $\$ 246$ \\
\hline \multicolumn{5}{|c|}{ Prior/Concurrent Conditions } \\
\hline \multicolumn{5}{|c|}{ Prior Vaccination } \\
\hline Yes & $\$ 1,264$ & $\$ 89$ & $\$ 1,453$ & $\$ 177$ \\
\hline No & $\$ 275$ & $\$ 68$ & $\$ 369$ & $\$ 100$ \\
\hline \multicolumn{5}{|c|}{ Recently Preceding Complication or Comorbidity Diagnosis } \\
\hline Yes & $\$ 776$ & $\$ 87$ & $\$ 944$ & $\$ 126$ \\
\hline No & $\$ 167$ & $\$ 66$ & $\$ 245$ & $\$ 93$ \\
\hline \multicolumn{5}{|c|}{ Concurrent Dispensing of Amantadine or Rimantadine } \\
\hline Yes & $\$ 177$ & $\$ 92$ & $\$ 320$ & $\$ 127$ \\
\hline No & $\$ 304$ & $\$ 67$ & $\$ 399$ & $\$ 100$ \\
\hline \multicolumn{5}{|c|}{ Prior Influenza Episode } \\
\hline Yes & $\$ 187$ & $\$ 76$ & $\$ 263$ & $\$ 106$ \\
\hline No & $\$ 306$ & $\$ 68$ & $\$ 404$ & $\$ 100$ \\
\hline \multicolumn{5}{|c|}{ Quintile of 3-Month Preceding Health Care Cost } \\
\hline $1: \$ 0$ & $\$ 124$ & $\$ 62$ & $\$ 161$ & $\$ 71$ \\
\hline $2:>\$ 0-\$ 86$ & $\$ 104$ & $\$ 62$ & $\$ 140$ & $\$ 75$ \\
\hline $3: \$ 87-\$ 199$ & $\$ 132$ & $\$ 62$ & $\$ 178$ & $\$ 86$ \\
\hline 4: $\$ 200-452$ & $\$ 225$ & $\$ 75$ & $\$ 288$ & $\$ 114$ \\
\hline $5: \$ 453+$ & $\$ 907$ & $\$ 100$ & $\$ 1,208$ & $\$ 211$ \\
\hline \multicolumn{5}{|c|}{ Evidence of Medication Use and Prevalent Comorbid Conditions } \\
\hline \multicolumn{5}{|c|}{ Chronic Corticosteroid Use } \\
\hline Yes & $\$ 1,505$ & $\$ 263$ & $\$ 1,763$ & $\$ 376$ \\
\hline No & $\$ 294$ & $\$ 68$ & $\$ 390$ & $\$ 100$ \\
\hline \multicolumn{5}{|l|}{ Malignancy } \\
\hline Yes & $\$ 5,180$ & $\$ 165$ & $\$ 5,904$ & $\$ 400$ \\
\hline No & $\$ 245$ & $\$ 68$ & $\$ 335$ & $\$ 100$ \\
\hline \multicolumn{5}{|c|}{ Chronic Heart Disease } \\
\hline Yes & $\$ 2,866$ & $\$ 162$ & $\$ 3,295$ & $\$ 334$ \\
\hline No & $\$ 248$ & $\$ 67$ & $\$ 338$ & $\$ 99$ \\
\hline \multicolumn{5}{|c|}{ Chronic Lung Disease } \\
\hline Yes & $\$ 510$ & $\$ 86$ & $\$ 637$ & $\$ 128$ \\
\hline No & $\$ 248$ & $\$ 66$ & $\$ 337$ & $\$ 94$ \\
\hline \multicolumn{5}{|l|}{ Diabetes } \\
\hline Yes & $\$ 1,949$ & $\$ 124$ & $\$ 2,290$ & $\$ 290$ \\
\hline No & $\$ 269$ & $\$ 68$ & $\$ 362$ & $\$ 100$ \\
\hline
\end{tabular}


age groups (55-64 and 65+) were nearly twice as likely to be hospitalized $(55-64 \mathrm{OR}=1.75 ; 95 \% \mathrm{CI}=0.87-3.52$; $65+\mathrm{OR}=1.93 ; 95 \% \mathrm{CI}=0.82-4.56)$, as were those in the 35-44 year old group. Patients with a recorded immunization were over 2.17 times as likely (95\% CI 1.06-4.43) to be hospitalized than patients without evidence of immunization. Intervals of care with recent influenza/ILIrelated complications or co-morbid diagnoses (within 30 days) were 3.2 times (95\% CI 1.96-5.13) more likely to be hospitalized compared to those without a recent concurrent complication. Episodes with the highest quintile of medical care costs in the preceding three months had about a 1.7 - fold increased likelihood of being hospitalized (95\% CI o.77-3.84). Prevalent co-morbid conditions and medication use were generally associated with an increased likelihood of hospitalization.

The logistic models for possible predictors of influenza/ ILI-related complications showed that children under the age of five years $(O R=1.55 ; 95 \% \mathrm{CI}=1.26-1.91)$ and adults over the age of 65 years were more likely (OR = 1.54; 95\% CI $=1.06-2.24)$ to experience complications when compared to the 35-44 age group. Intervals of care for wpatients diagnosed with recent influenza/ILI-related complications or co-morbid diagnosis (30 days prior) were more likely $(\mathrm{OR}=1.47 ; 95 \% \mathrm{CI} 1.27-1.70)$ to result in patients having influenza/ILI-related complications. Higher quintiles for cost of medical services received in the 3-month period preceding the influenza/ILI diagnosis were more strongly associated with complications of influenza/ILI ( $4^{\text {th }}$ quintile OR $=1.21 ; 95 \% \mathrm{CI}=1.00-$ $1.47 ; 5^{\text {th }}$ quintile OR $\left.=1.56 ; 95 \% \mathrm{CI}=1.28-1.91\right)$. Prevalent co-morbidities such as maligna (95\% CI = 1.19-2.61) and diabetes $(\mathrm{OR}=1.74 ; 95 \% \mathrm{CI}=1.16-2.61)$ were significantly related to the occurrence of influenza/ILI-related complications during intervals of care for influenza/ILI. (Table 3)

Table 4 shows the total, mean and median costs incurred on influenza/ILI diagnosis date and during the 21-day follow-up period, stratified by possible predictor variables. Intervals of care for patients with prevalent co-morbid conditions had a consistently higher median influenza/ILI-related costs compared to those without co-morbidities. Influenza/ILI-related costs accounted for $76 \%$ of the total health care costs incurred during the interval of care $(\$ 1,981,072 / \$ 2,622,179)$.

\section{Discussion}

Elderly patients and patients with an underlying chronic illness, a recent complication, or a higher health care cost in the recent months were at the highest risk of hospitalization and complications after a diagnosis of influenza/ ILI. Influenza/ILI-related health care costs represented the bulk of total health care costs during an influenza/ILI interval of care. In addition to a confirmation of what we would expect qualitatively for the healthcare burden of influenza, our observation provides a useful quantitative estimate for these effects in the general population.

The diagnosis of influenza/ILI was based on ICD-9 codes and did not incorporate viral isolates or laboratory confirmation. "Influenza/ILI" in this study is likely to be representative, on a population level, of influenza/ILI diagnoses and a smaller percentage of more severe influenza-like illness. The 21-day follow-up period incorporated a clinical expectation that the vast majority of influenza/ ILI-related complications would occur within this period, and that complications that extended beyond 21 days would usually be ascribable in part to pre-existing illness. More sophisticate approaches are imaginable, but not among multiple causes, which in any case would have been impossible to implement at the level of insurance claims analysis.

This study found that patients with a record of an influenza immunization were at a higher risk for hospitalization than those who were not vaccinated. This is clearly not a vaccine effect, but instead an effect attributable to the kinds of patients in the United States who typically received influenza vaccination in a doctor's office during the period of the study. Vaccine is indicated for patients at high risk of complications, and that risk applies just as much to ILI, which presumably account for the great majority of events among vaccinated persons. Antiviral use was not extensive, and the current study dates preceded the introduction of zanamivir and oseltamivir. It appears that zanamivir reduces the risk of complication in highrisk patients. [12] We found some indication (although not statistically significant) that use of amantadine and rimantadine may have prevented hospitalizations $(\mathrm{OR}=$ o.6) and substantially reduced costs.

Influenza/ILI adversely affects individuals with a variety of chronic underlying conditions, supporting the Center for Disease Control and Prevention Immunization recommendations for influenza immunization [15]. Unlike other studies that have evaluated the cost-effectiveness of influenza immunization [13-16], our data quantify a major cost impact of ILI and influenza/ILI in a large population (over 6200 patients) with wide-variation in ages and health status. These data may be useful in understanding the patient groups at high risk for influenza-related complications and assisting the cost-benefit evaluation of a more universal influenza immunization program for these patients.

Both chronic and acute manifestations of comorbid illness were strong, independent predictors of influenza/ ILI-related complications and hospitalizations. Addi- 
tionally, health care costs in the three months before the influenza/ILI episode serve as a surrogate for the overall health status of a patient and could reflect the severity of the underlying diseases. We were unable to evaluate the effect of HIV infection, chronic renal disease, and immunosuppressive therapy due to small number of patients with these co-morbidities. These groups of patients are at high risk for influenza/ILI-related complications and health care costs. Therefore, our estimates are most likely an under representation of the true burden of influenza/ILI.

The 21-day follow-up period incorporated a clinical expectation that the vast majority of influenza/ILI-related complications would occur within this period, and that complications that extended beyond 21 days would usually be ascribable in part to preexisting illness. We chose the 21-day cutoff as a rough-and-ready, approximate partition of mulitfactorial disease burden. More sophisticate approaches are imaginable, but not among multiple causes, which in any case would have been impossible to implement at the level of insurance claims analysis.

These data reflect patterns of care for influenza/ILI seen within the NEI health care system. NEI beneficiaries who sought medical care outside the NEI system would not be captured in the claims data and are not included in this analysis. This is probably a relatively small percentage of patients and is unlikely to bias the results. More importantly, care for many respiratory conditions (especially otitis media and acute bronchitis) was captured in this study only as a complication of previously recognized influenza/ILI. If the original illness had been diagnosed only as one of these conditions, it would never have entered into the study. In addition, we lack data on patients who did not seek medical care for influenza/ILI. Medications purchased outside the insurance plan (e.g. overthe-counter) are not included in the database. Thus, influenza/ILI-related costs we report are likely to be an under estimate of the true costs of medical care, and this is only a fraction of the total societal cost, which would include at a minimum lost wages and productivity. NEI enrolls relatively few persons over the age of 65 , due to the availability of Medicare coverage for the elderly. The elderly in NEI include those who are still working or who formerly worked for the state or federal government and therefore are not eligible for Medicare.

Approximately $78 \%$ of influenza isolates from the World Health Organization Collaborating Laboratories were type A and 22\% were type B during the 1996 - 1997 influenza season. Type A influenza is thought to cause more severe illness [17-19]. Therefore, the majority of influenza/ILI diagnoses captured in the NEI system us- ing ICD-9 codes would be expected to be type A and represent a higher proportion of true influenza illness than ILI during the 1996-1997 influenza season.

Antiviral use was not extensive, and the current study dates preceded the introduction of zanamivir and oseltamivir. We found some indication (although not statistically significant) that antiviral use may have prevented hospitalizations $(\mathrm{OR}=0.6)$. The recent marketing of influenza antivirals may have an even greater impact on the predictors of complications and health care related costs of influenza.

Strengths of this study include the large cohort of ILI and influenza cases (over 6200 patients) and the diversity of the risk profiles for influenza/ILI-related complications in this population. These data should be applicable to other large health maintenance organizations and would be useful for evaluating the burden of influenza/ILI in these populations.

\section{Conclusions}

Recent acute events foretell complications of influenza, as does underlying chronic illness. Most costs in the three weeks following onset are for influenza/ILI-related care.

\section{Competing interests}

$\mathrm{SC}, \mathrm{W}-\mathrm{YH}$, and DR were employees of Glaxo Wellcome (now GlaxoSmithKline), the manufacturer of zanamivir, at the time this study was carried out. DI, AMW, and LW were employees of lngenix Pharmaceutical Services, which received a research contract to perform this study from Glaxo Wellcome.

\section{Additional material}

\section{Supplementary files}

Appendix A: ICD-9 Codes for influenza-Related Complications and Manifestations.Contents are as name implies.

Appendix A.doc

[http://www.biomedcentral.com/content/supplementary/14726963-1-8-S1.doc]

Appendix B.doc

[http://www.biomedcentral.com/content/supplementary/14726963-1-8-S2.doc]

\section{References}

I. Simonsen L, Clarke MJ, Williamson GD, Stroup D, Arden NH, Schonberger LB: The Impact of influenza epidemics on mortality: introducing a severity index. Am J Public Health 1997, 87: $1944-1950$

2. Barker WH: Excess pneumonia and influenza-associated hospitalization during influenza epidemics in the U.S., 19701978. Am J Public Health 1986, 76:761-765 
3. Centers for Disease Control and Prevention: Prevention and control of influenza: recommendations of the Advisory Committee on Immunization Practices (ACIP). MMWR 2000, 49(No. RR-3):

4. Centers for Disease Control and Prevention: Prevention and control of influenza: recommendations of the Advisory Committee on Immunization Practices (ACIP). MMWR 1998, 47(RR-6): I-26

5. Perotta DM, Decker M, Glezen WP: Acute respiratory disease hospitalizations as a measure of impact of epidemic influenza: Am J Epidemiol I 985, I 22:468-476

6. Nichol K: Complications of influenza and benefits of vaccination. Vaccine 1999, 17:S47-S52

7. McBean A, Babish J, Warren J: The impact and cost of influenza in the elderly. Arch Intern Med 1993, 153:2105-21II

8. International Classification of Diseases, 9 th Revision, Clinical Modification. 5th edition. Medicode Publications, Salt Lake City UT, 1997

9. American Medical Association: Physicians' Current Procedural Terminology: CPT 97. American Medical Association, Chicago IL, 1997

10. HCPCS: Medicare's National Level II Codes. Medicode Publications, Salt Lake City UT, 1997

II. AHFS 97: Drug Information. American Hospital Formulary Service, Bethesda MD, 1997

12. The MIST (Management of Influenza in the Southern Hemisphere Trialists) Study Group: Randomised trial of efficacy and safety of inhaled zanamivir in treatment of influenza $A$ and $B$ virus infections. Lancet 1998, 352:|877-8।

13. Mullooly J, Bennett M, Hornbrook M, Barker W, Williams W, Patriarca $\mathrm{P}$, Rhodes $\mathrm{P}$ : Influenza vaccination programs for elderly persons: cost effectiveness in a health maintenance organization. Ann Intern Med 1994, I 2 1:947-952

14. Nichol K: Complications of influenza and benefits of vaccination. Vaccine 1999, 17:S47-S52

15. Postma M, Bos J, van Gennep M, Jager J, Baltussen R, Sprenger M: Economic evaluation of influenza vaccination. Assessment for the Netherlands. Pharmacoeconomics 1999, 16 Suppl 1:33-40

16. Nichol K, Margolis K, Wuorenma J, Von Sternberg T: The efficacy and cost effectiveness of vaccination against influenza among elderly persons living in the community. NEJM 1994 , 331:778-784

17. World Health Organization. Weekly Epidemiology Record 1998, 73(7):4I-48

18. Centers for Disease Control and Prevention: Surveillance for Influenza - United States, 1994-95, 1995-96, and 1996-97 Seasons. MMWR 2000, 49(SS03): I3-28

19. Mandell G, Bennett J, Dolin R: Principles and Practice of Infectious Disease 4th Edition, Churchill Livingstone 1995

\section{Pre-publication history}

The pre-publication history for this paper can be accessed here:

http://www.biomedcentral.com/content/backmatter/ 1472-6963-1-8-b1.pdf

Publish with BioMed Central and every scientist can read your work free of charge

"BioMedcentral will be the most significant development for disseminating the results of biomedical research in our lifetime."

Paul Nurse, Director-General, Imperial Cancer Research Fund

Publish with BMC and your research papers will be:

- available free of charge to the entire biomedical community

- peer reviewed and published immediately upon acceptance

- cited in PubMed and archived on PubMed Central

- yours - you keep the copyright

Submit your manuscript here:

http://www.biomedcentral.com/manuscript/
BioMedcentral.com editorial@biomedcentral.com 